Sharif University of Technology
Scientia Iranica
Transactions E: Industrial Engineering
SCIENTIA
I RAN I C A

\title{
A new mathematical model for integrated production planning and scheduling problem in capacitated flexible flow shop with sequence-dependent setups
}

\author{
R. Ramezanian ${ }^{\mathrm{a}, *}$, S. Fallah Sanami ${ }^{\mathrm{b}}$ and V. Mahmoodian ${ }^{\mathrm{c}}$ \\ a. Department of Industrial Engineering, K.N. Toosi University of Technology, Tehran, Iran. \\ b. Department of Economic and Management, Semnan University, Semnan, Iran. \\ c. Department of Industrial Engineering, Iran University of Science and Technology, Tehran, Iran.
}

Received 15 June 2015; received in revised form 29 June 2016; accepted 22 August 2016

\author{
KEYWORDS \\ Lot-sizing and \\ scheduling; \\ Flexible flow shop; \\ Mathematical model; \\ Sequence-dependent \\ setup; \\ MIP-based method; \\ Fix-and-relax \\ procedure.
}

\begin{abstract}
The main contribution and novelty of this paper is proposing a more efficient mathematical model for integrated lot-sizing and scheduling in a multi-product multiperiod capacitated flexible flow shop with sequence-dependent setups. A new approach for modeling the problem has been proposed and its complexity has been compared with former models. In comparison to the former models, fewer continuous and binary variables and constraints of the proposed model make it easy to solve. Comparison between the new model and the former models proves the superiority of the proposed model. Due to complexity of the problem, three mixed-integer programming based heuristics, all based on iterative resolutions of reduced-size MIPs and rolling horizon, have been implemented to solve the model. To evaluate the performance of the proposed model and solution method, problems of different scales have been studied. The used algorithms search the solution space for both lot-sizing and scheduling and find a combination of production planning and scheduling that is feasible and close to optimum. Computational results show that HA2 is superior for this problem and can find good quality solution to it in a reasonable computational time. Also, sensitivity analysis is used to clarify the problem and ensure suitability of the proposed model.

(C) 2017 Sharif University of Technology. All rights reserved.
\end{abstract}

\section{Introduction}

Every organization needs to provide high quality services and low prices. Lack of attention to each of these parameters weakens the competition ability of organizations and causes them to lose their life. The factors influencing the quality and price of services

\footnotetext{
*. Corresponding author. Tel.:+98 2184063365 ;

Fax: +982188674858

E-mail addresses: Ramezanian@kntu.ac.ir (R.

Ramezanian); Fallah.Sahar@gmail.com (S. Fallah Sanami); vahid_mahmoodian@ind.iust.ac.ir (V. Mahmoodian)
}

doi: $10.24200 /$ sci. 2017.4389 and goods determine the production rate and the time of services. Companies should fulfill customer orders on time and if orders are not fulfilled on time, they lose a lot of profit and for achieving this purpose, lotsizing and scheduling are very essential. Lot-sizing and scheduling are a part of the operative production planning system. Lot-sizing problem is considered as an input to scheduling problem and these problems are closely related to each other. In lot sizing, a lot shows the quantity of an item produced on a machine continuously without interruption. The aim of scheduling is to determine the sequence of operations that are performed on a machine $[1,2]$.

Production planning and scheduling are the most 
important issues of the production industries, which have a considerable influence on the productivity of the production systems. In traditional scheduling problems, the production operations are considered regardless of detailed scheduling decisions. It is very important to identify that scheduling and lot-sizing are not independent and they should be integrated and determined simultaneously. In our paper, we address joint lot-sizing and scheduling in a multi-product multiperiod capacitated flexible flow shop manufacturing system with sequence-dependent setups. As you know, flexible flow shop is one of the modern manufacturing systems fairly common in flexible manufacturing and process industry. This problem has many applications in assembly workshops such as automobile assembly lines [3], automotive parts manufacturing industry, assembly lines of household electric appliances, assembling workshop in an auto body plant, tile industry [4], printed circuit board manufacturing system [5], etc. For example, automobile assembly lines consist of determining lot-sizes and sequences of automobiles, such as buses, trucks, and cars, to be assembled.

Since Wagner and Whithin [6] published their seminal paper on lot-sizing and scheduling problems in 1958, they have remained an active area of research for contributors. After presenting this basic paper, researchers have done a lot of studies to formulate and provide efficient solution algorithms for lot-sizing and scheduling problems.

The models presented for lot-sizing and scheduling are classified into small-bucket and big-bucket problems [7]. "Big-Bucket" problems have long time periods in which several products can be manufactured and "Small-Bucket" problems break the planning horizon into smaller time periods, which limit the number of items that can be produced in a single period. Thus, lot-sizing and scheduling decisions can be made simultaneously using these models. The interested reader to small-bucket problems is referred to Tempelmeier and Buschkuhl [8] for further information.

Buschkühl et al. [9] reviewed the studies over the past four decades on Multi-Level Capacitated LotSizing Problems (MLCLSP). Small-bucket problems for multi-level production environment include MultiLevel Discrete Lot-Sizing and Scheduling Problem (MLDLSP) [10], Multi-Level Proportional Lot Sizing and Scheduling Problem (MLPLSP) [11,12]. MLDLSP and MLPLSP models enable simultaneous lot-sizing and scheduling, but limit the number of products that can be produced in a period. The Multi-Level Capacitated Lot-Sizing Problem (MLCLSP) is a bigbucket problem in which several products can be manufactured in a given period, but it cannot determine lot-sizes and schedules simultaneously. Fandel and Stammen-Hegene [1] proposed Multi-Level General Lot-Sizing and Scheduling Problem (MLGLSP) based on two-level time structure [13] and tried to integrate the advantages of MLPLSP and MLCLSP. MLGLSP enables simultaneous lot-sizing and scheduling for multi-product multi-level job shop production. Due to the high computational complexity of the model, only very-small-size problems with a low number of products and periods can be solved optimally. Ponnambalam and Mohan Reddy [14] proposed a hybrid search algorithm by combining genetic algorithm and simulated annealing to minimize the maximum completion time of tasks and maintenance and overtime costs for lot-sizing problems and sequence. As mentioned, Fandel and Stammen-Hegene [1] studied simultaneous lot-sizing and scheduling problem for multi-product multi-level production system to minimize sequencedependent setup cost, inventory cost, production cost, and the cost to maintain the condition of setups. Toledo et al. [15] addressed the Synchronized and Integrated Two-Level Lot-sizing and Scheduling Problem (SITLSP). They proposed a mathematical model to formulate the problem and used an optimization package to solve a set of instances for this problem based on the data provided by a soft drink company.

Integrated lot-sizing and scheduling in a capacitated flow shop environment with sequencedependent setups has been considered by Mohammadi et al. [16,17] and Ramezanian et al. [2,18-20]. Mohammadi et al. [16,17] proposed a mathematical formulation and Mixed-Integer Programming (MIP)based solution methods for the problem. Mohammadi et al. [21] presented an algorithmic approach to solve large-size instances of the problem. Mohammadi et al. [22] used a genetic algorithm to solve their model for simultaneous lot-sizing and scheduling problem in the permutation flow shop with sequence-dependent setup. Ramezanian et al. [2] proposed a more efficient mathematical model and MIP-based heuristics for the problem of integrating lot-sizing and scheduling with sequence-dependent setups in flow shop environment. Ramezanian et al. [18,19] considered multi-product and multi-period integrated lot-sizing and scheduling problem for multi-stage environments with new operation conditions, machine unavailability, and operation overlapping, simultaneously. Later, Ramezanian and Saidi-Mehrabad [20] studied the mentioned problem with uncertain processing times and uncertain demand of various products. They implemented a hybrid simulated annealing and firefly algorithm to solve the problem.

Mohammadi et al. [16,17,21,22] and Ramezanian et al. [2,18-20] assumed that there is one machine that performs all the processing for each stage. Recently, Mohammadi [23] and Mohammadi and Jafari [24] ignored this limiting assumption. They assumed that at least one of the stages must have more than one machine. This extension transformed the flow shop 
into a flexible one. A flexible flow shop is one of the most usual production systems in manufacturing discrete parts that can be considered as a generalization of the flow shop and the parallel machine environments. This production environment involves serially arranged work centers where at least one of the production stages must have parallel machines. Products have the same sequence of operations in all stages [21,23]. Mohammadi [23] used the generalized model proposed by Fandel and StammenHegene [1] to formulate simultaneous lot-sizing and scheduling in flexible flow shop. In his approach, every planning period was decomposed into smaller periods so that in each of the micro-periods, a product was manufactured. Due to the large number of decision variables and constraints, this approach drastically increased the computational complexity of the problem. A more efficient method that was presented by Mohammadi and Jafari [23] to formulate the problem came out from developing the modeling approach, using an artificial setup concept of Clark [25] and Clark and Clark [26], which assumed that during every planning period, $N$ (number of products) setups occurred. But, as it is obvious, this presumption also increases the computational complexity of the problem. Babaei et al. [27] studied simultaneous lot-sizing and scheduling in flow shop with sequence-dependent setup, setup carry over, and backlogging. They developed a genetic algorithm to solve the problem. Urrutia et al. [28] investigated multi-item multi-period multi-resource single-level lotsizing and scheduling problems in job-shop environments with capacity constraints. They proposed a solution method based on a Lagrangian heuristic that determined a feasible production plan for a fixed schedule. Their approach iteratively fed the heuristic with a sequential improvement. Wolosewicz et al. [29] presented a new mathematical model for generating a feasible optimal production plan for a fixed schedule on the machines considering capacity constraints and setup decisions. They proposed a novel constructive heuristic based on Lagrangian relaxation for solving the problem. Karimi-Nasab et al. [30] considered lot sizing and scheduling problem with compressible process times in a job-shop environment. They used Branch and Cut (B\&C) approach to solve the problem and added some valid inequalities to it. KarimiNasab et al. [31] presented a model formulation for a multi-level lot sizing problem with flexible machines, which included a job-shop problem. They developed a Particle Swarm Optimization (PSO) algorithm to solve the problem. Yan et al. [32] proposed an iterative genetic algorithm for integrated production planning and scheduling optimization problem in a job shop environment. Rohaninejad et al. [33] presented a multi-stage GLSP formulation for a flexi- ble job-shop problem. They used a hybrid metaheuristic based on the combination of a Genetic Algorithm (GA) and a PSO to solve the problem. For more details, the interested readers are referred to the review paper that has been published recently [34].

Schemeleva et al. [35] addressed a stochastic multi-product lot-sizing and sequencing problem with two kinds of uncertainties, including defective items due to the machines' imperfections and random lead time and sequence-dependent set-up times. Masmoudi et al. [36] considered a capacitated lot-sizing problem with energy consideration in flow shop manufacturing system. They proposed non-linear and linear mixedinteger programming models to formulate the problem.

We propose a more efficient Mixed-Integer Programming (MIP) model to formulate the problem, which can simultaneously achieve a production plan and schedule. In comparison with the models presented by Mohammadi [23] and Mohammadi and Jafari [24], presenting a more efficient mathematical model for integrated lot-sizing and scheduling in capacitated flexible flow shop production system with sequencedependent setups is the main novelty of this paper. In the literature, MIP-based heuristics are one of the appropriate approaches that have been suggested for solving dynamic lot-sizing and scheduling problem. As a result, less complexity of the proposed model results in more effectiveness of the solution methods. Also, three MIP-based heuristics, all based on iterative resolutions of reduced-size MIPs and rolling horizon, have been implemented to solve the problem.

This paper has the following structure. Section 2 provides a detailed description of the new mathematical model, underlying assumptions, and comparison with the former models (provided by Mohammadi [23] and Mohammadi and Jafari [24]). Section 3 provides heuristic approaches to solve the considered problem. Section 4 reports the numerical experiments and, finally, Section 5 discusses the concluding remarks and recommendations for future research.

\section{Problem formulation}

The main novelty of this paper is proposing a more efficient mathematical model for the problem of integrating lot-sizing and scheduling in capacitated flexible flow shop environments. This section contains the description of the proposed MIP model with more details and the comparison between the new model and two former models.

\subsection{Assumptions}

Our assumptions are similar to those of Mohammadi [23] and Mohammadi and Jafari [24]. The main assumptions are listed as follows: 
- Lot-sizing and scheduling are performed simultaneously;

- Several products can be produced in serially arranged stages;

- At least one stage has parallel machines and machines in each stage are identical in all characteristics such as production rates, setup times, and costs;

- At stages with more than one machine, each product is produced entirely on one machine;

- Machines in stage $m$ can only process the intermediary products of the $m$ 'th level that are required for production of the product type of level $m+1$;

- Each machine is constrained in capacity;

- Setting up of a certain item incurs a setup time and relevant setup cost and it is sequence-dependent. Setup follows the so-called triangular inequalities, i.e. it is never faster to change over from one product to another by means of a third product;

- Setup costs have the form $W_{i j m}=f_{w} . S_{i j m}$ where $f_{w}$ is opportunity cost per unit of setup time;

- The setting up of a machine must be completed in a period;

- The required resources and parts must be ready for production;

- External demands for the final products are known and deterministic, and are satisfied at the end of each period;

- There are no lead times between the different production stages for transportation or cooling of the products;

- Shortages are not permitted;

- A component cannot be produced in a period until the production of its required components is finished. In other words, production in a production stage can only be started if all amounts of the required items from the previous production stage are available; this is called vertical interaction;

- At the beginning of the planning horizon, machines are set up for a defined product;

- At any time, each machine can process at most one job;

- At any time, each job can be processed on at most one machinel;

\subsection{Former models}

Here, to shorten the length of the paper, we avoid presenting the details of the former models and interested readers can refer to Mohammadi [23] and Mohammadi and Jafari [24].

In the mathematical model presented by Mohammadi [23], the two-level time structure [1,13] has been used to formulate the mentioned problem. The planning horizon is divided into $T$ macro-periods with the same length so that in each of the micro-periods, a product is manufactured. For each machine, each macro-period, $t$, consists of $3 N$ micro-periods ( $N$ is the number of products). As it is obvious, due to the large number of decision variables and constraints, this approach drastically increases the computational complexity of the model. A more efficient method that was presented by Mohammadi and Jafari [24] uses artificial setup concept to formulate this problem similar to those of Clark [25] and Clark and Clark [26]. There must be precisely $N$ (number of products) setups in each period on each machine, even if a setup is just from a product to itself. But, as it is obvious, this presumption also increases the complexity of the problem. Also, by introducing the shadow products, the number of variables increases $\left(N^{2} \cdot T \cdot \sum_{m=1}^{M} n_{m}\right)$. Note that these assumptions are not needed in the new model proposed in this paper.

MIP-based heuristics are one of the appropriate approaches that have been suggested in the literature for solving dynamic lot-sizing and scheduling problem. As a result, less complexity of the proposed model results in more effectiveness of the solution methods. Therefore, in this paper, a new approach is proposed to formulate lot-sizing and scheduling problem in a multi-stage production environment with parallel machines, which considerably reduces the complexity of the model.

\subsection{New model}

In this subsection, MIP model is proposed for lot sizing and scheduling problem in a multi-product, multiperiod production system in a flexible flow shop with capacity constraint and sequence-dependent setup. Simultaneous lot-sizing and scheduling problem in a flexible $M$-stage flow shop involves determining the production quantity and sequence of $n$ jobs in the series systems that have more than one machine for processing at least in one stage. Jobs are subject to predecessor constraints that restrict them to have a certain process plan in all stages of operation. Therefore, a product contains $M$ operations, one for each stage of production, and facilities (machines) have capacity constraints.

In order to formulate the model, the following data has been used:

\section{Indices}

$i, j \quad$ Indices of product types

$m \quad$ Stage index

$l \quad$ Machine index

$t \quad$ Planning period index 


\section{Parameters}

$T \quad$ Planning horizon (in terms of the number of planning periods) (set of periods)

$N \quad$ Number of different products (set of product types)

$M \quad$ Number of stages (set of stages)

$n_{m} \quad$ Number of parallel machines in stage $m$

$C_{m t} \quad$ Processing time (capacity) available for each machine at stage $m$ in period $t$ (in time units)

$d_{j t} \quad$ External demand for the $j$ 'th product at the end of period $t$

$b_{j m} \quad$ Required capacity of machine at stage $m$ to produce one unit of product $j$ (in time units)

$P_{j m t} \quad$ Production cost per unit of finished product $j$ processed on machine at stage $m$ in period $t$

$h_{j m} \quad$ Holding cost per unit of finished product $j$ at stage $m$

$S_{i j m} \quad$ Sequence-dependent setup time of switching from product $i$ to $j$ on machine at stage $m$

$w_{i j m} \quad$ Sequence-dependent setup cost of switching from product $i$ to $j$ on machine at stage $m$

$I_{j 0} \quad$ Inventory of product $j$ at the beginning of planning horizon

BigM A large real number

\section{Decision variables}

$X_{j m t} \quad$ Quantity of product $j$ produced at stage $m$ in period $t$

$I_{j m t} \quad$ Inventory of finished product $j$ at stage $m$ in period $t$

$S O_{m l j t} \quad$ Starting time of product $j$ on machine $l$ at stage $m$ in period $t$

$C O_{m l j t}$ Completion time for product $j$ on machine $l$ at stage $m$ in period $t$

$Z_{j m t} \quad$ A binary variable that is equal to 1 if $X_{j m t}>0,0$ otherwise

$Q_{m l j t} \quad$ A binary variable that is equal to 1 if job $j$ is assigned to process on machine $l$ at stage $m$ in period $t, 0$ otherwise

$Y_{\text {mlijt }} \quad$ A binary variable that is equal to 1 if job $j$ is processed immediately after job $i$ on machine $l$ at stage $m$ in period $t, 0$ otherwise

\section{Mixed-integer programming model}

In this subsection, the proposed MIP model is presented with more details:

$$
\begin{aligned}
\operatorname{Min} T C= & \sum_{j=1}^{N} \sum_{m=1}^{M} \sum_{t=1}^{T} p_{j m t} \cdot X_{j m t} \\
& +\sum_{j=1}^{N} \sum_{m=1}^{M} \sum_{t=1}^{T} h_{j m} \cdot I_{j m t} \\
& +\sum_{i=1}^{N} \sum_{j=1}^{N} \sum_{m=1}^{M} \sum_{l=1}^{n_{m}} \sum_{t=1}^{T} w_{i j m} . Y_{m l i j t},
\end{aligned}
$$

s.t.

$$
\begin{gathered}
I_{j M(t-1)}+X_{j M t}=I_{j M t}+d_{j t} \quad \forall j, t, \\
I_{j m(t-1)}+X_{j m t}=I_{j m t}+X_{j(m+1) t}, \\
\forall j, t, m=1, \ldots, M-1, \\
X_{j m t} \leq b i g M . Z_{j m t} \quad \forall m, j, t, \\
\sum_{l=1}^{n_{m}} Q_{m l j t}=Z_{j m t} \quad \forall m, j, t,
\end{gathered}
$$$$
C O_{m l j t} \leq C_{m t} \quad \forall m, l, j, t,
$$$$
\sum_{l=1}^{n_{m}} C O_{m l j t} \cdot Q_{m l j t}=\sum_{l=1}^{n_{m}} S O_{m l j t} \cdot Q_{m l j t}
$$$$
+\sum_{l=1}^{n_{m}} b_{j m} \cdot X_{j m t} \cdot Q_{m l j t} \quad \forall m, j, t,
$$

$$
\sum_{l=1}^{n_{m}} S O_{m l j t} \cdot Q_{m l j t} \geq \sum_{l=1}^{n_{m}} C O_{(m-1) l j t} \cdot Q_{(m-1) l j t}
$$

$$
\forall j, t, m=2, \ldots, M,
$$

$$
\begin{aligned}
S O_{m l j t} \cdot Q_{m l j t} \geq C O_{m l i t} \cdot Q_{m l i t}+S_{i j m} \cdot Y_{m l i j t} \\
-b i g M .\left(1-Y_{m l i j t}\right) \quad \forall m, l, i, j, t, i \neq j,
\end{aligned}
$$

$\sum_{i=1}^{N} \sum_{\substack{j=1 \\ i \neq j}}^{N} Y_{m l i j t} \geq \sum_{j=1}^{N} Q_{m l j t}-1 \quad \forall m, j, t$,

$\sum_{\substack{i=1 \\ i \neq j}}^{N} Y_{m l i j t} \leq Q_{m l j t} \quad \forall m, l, j, t$

$\sum_{\substack{i=1 \\ i \neq j}}^{N} Y_{m l j i t} \leq Q_{m l j t} \quad \forall m, l, j, t$, 


$$
\begin{aligned}
& I_{j m 0}=0 \quad \forall m, j, \\
& X_{j m t}, I_{j m t}, S O_{m l j t}, C O_{m l j t} \geq 0 \quad \forall m, l, j, t, \\
& Y_{m l i j t}, Z_{j m t}=\{0,1\} .
\end{aligned}
$$

The objective function (Constraint 1 ) is to minimize the sum of the production cost, holding cost, and sequence-dependent setup costs. Constraint (2) ensures the demand supply for each product in each period. Constraint (3) is the usual flow balance constraints and shows that in a network, the total inflows to each node are equal to out-flows from that node. Constraint (4) shows the relationship between the productions planned and the binary variable indicating that product $j$ at stage $m$ in period $t$ is produced or not. This constraint states that if $X_{j m t}>0$, variable $Z_{j m t}$ takes the value 1 automatically. Constraint (5) determines the assigning of products to the machines at each stage of production. Constraint (6) represents the capacity constraints of machines during periods. Constraint (7) shows the relationship between the start time and completion time of process for products that are manufactured in each period. Constraint (9) forces to start the processing of each product only when its precedent product has been completed on the same machine at the stage of production in addition to its setup time. Constraints (10)-(12) determine the sequence of products in a hybrid flow shop production environment. According to the Constraints (11) and (12), job sequence includes product $j$ if it is produced in period $t$ and assigned to machine $l$ at stage $m$. Constraint (13) indicates that at the beginning of planning horizon, there is no on-hand inventory. Constraints (14) and (15) represent the types of decision variables.

Values for coefficients and parameters of the model can be easily obtained from actual data of a plant or can be estimated through historical data acquisition.

\subsection{Comparison between new and former models}

Herew, the proposed model is compared with the models of Mohammadi [23] and Mohammadi and Jafari [24]. Table 1 shows the comparison between former models and the new model. A number of binary variables, number of continuous variables, and number of constraints have been used in this comparison. Table 2 shows numerical examples that compare former and proposed models in specific problem sizes with $\left(N, M, n_{m}, T\right)=(5,3,2,6)$ and $\left(N, M, n_{m}, T\right)=$ $(25,5,3,12)$. According to Table 2, the number of continuous and binary variables and the number of constraints for the proposed model are remarkably less than those for Mohammadi and Jafari [24]. It should be noted that the proposed modeling approach

\begin{tabular}{|c|c|c|c|}
\hline Criterion & $\begin{array}{c}\text { Mohammadi's } \\
\text { model [20] }\end{array}$ & $\begin{array}{c}\text { Mohammadi \& } \\
\text { Jafari's model [24] }\end{array}$ & Proposed model \\
\hline $\begin{array}{c}\text { Number of } \\
\text { binary variables }\end{array}$ & $3 N^{2} T(N+2) \sum_{m=1}^{N} n_{m}$ & $N^{3} T \sum_{m=1}^{M} n_{m}$ & $N T\left(M+(1+N) \sum_{m=1}^{M} n_{m}\right)$ \\
\hline $\begin{array}{c}\text { Number of } \\
\text { continuous variables }\end{array}$ & $N T\left(M+6 N \sum_{m=1}^{M} n_{m}\right)$ & $N T\left(M+2 N \sum_{m=1}^{M} n_{m}\right)$ & $2 N T\left(M+\sum_{m=1}^{M} n_{m}\right)$ \\
\hline $\begin{array}{l}\text { Number of } \\
\text { constraints }\end{array}$ & $\begin{array}{l}9 N^{3} T \sum_{m=1}^{M-1}\left(n_{m} n_{m+1}\right. \\
\quad+N T\left(3 N^{3}-6 N^{2}+9 N+3\right) \\
\quad \sum_{m=1}^{M} n_{m}+N\left(1-N^{2}+2 N\right) \\
\quad \sum_{m=1}^{M} n_{m}+2 N M T\end{array}$ & $\begin{array}{l}N^{3} T \sum_{m=1}^{M-1}\left(n_{m} n_{m+1}\right) \\
\quad+\left(3 N^{2} T+N^{2}+T-2 N+1\right) \\
\quad \sum_{m=1}^{M} n_{m}+N M(1+2 T)\end{array}$ & $\begin{array}{l}N T(2+N) \sum_{m=1}^{M} n_{m} \\
\quad+N M(1+6 T)-N T\end{array}$ \\
\hline
\end{tabular}
has resulted in a nonlinear model. To reduce the

Table 1. A comparison between former models and the proposed model.

\begin{tabular}{|c|c|c|c|c|c|c|}
\hline & \multicolumn{3}{|c|}{$N=5, M=3, n_{m}=2, T=6$} & \multicolumn{3}{|c|}{$N=25, M=5, n_{m}=3, T=12$} \\
\hline & $\begin{array}{c}\text { Mohammadi \& } \\
\text { Jafari's } \\
\text { model }[24] \\
\end{array}$ & $\begin{array}{l}\text { Proposed } \\
\text { model }\end{array}$ & Difference & $\begin{array}{c}\text { Mohammadi \& } \\
\text { Jafari's } \\
\text { model }[24] \\
\end{array}$ & $\begin{array}{c}\text { Proposed } \\
\text { model }\end{array}$ & Difference \\
\hline Number of binary variables & 4,500 & 1,170 & 3,330 & $2,812,500$ & 118,500 & $2,694,000$ \\
\hline Number of continuous variables & 1,890 & 540 & 1,350 & 226,500 & 12,000 & 214,500 \\
\hline Number of constraints & 9,027 & 1,785 & 7,242 & $7,099,445$ & 130,325 & $6,969,120$ \\
\hline
\end{tabular}

Table 2. A comparison between former models and the proposed model for two different problem sizes. 
computational complexity, the model should be linearized; the linearization method is presented as follows.

As it is obvious, Constraint sets (7)-(9) are nonlinear terms that greatly increase the computational complexity for solving the problem. This constraint is modified in order to analyze it more easily. In more details, by removing the nonlinear Constraints (7)-(9), the set of simple linear Constraints (16)-(18) is replaced and Constraints (19)-(28) are added to the proposed model. Products $S O_{m l j t} \times Q_{m l j t}, C O_{m l j t} \times Q_{m l j t}$, and $X_{j m t} \times Q_{m l j t}$ can be replaced with additional continuous variables $S Q_{m l j t}, C Q_{m l j t}$, and $X Q_{m l j t}$, respectively. The following constraints must be added to force $S Q_{m l j t}, C Q_{m l j t}$, and $X Q_{m l j t}$ to take the values of $S O_{m l j t} \times Q_{m l j t}, C O_{m l j t} \times Q_{m l j t}$, and $X_{j m t} \times Q_{m l j t}$, respectively [37]:

$$
\sum_{l=1}^{n_{m}} C Q_{m l j t}=\sum_{l=1}^{n_{m}} S Q_{m l j t}+\sum_{l=1}^{n_{m}} b_{j m} \cdot X Q_{m l j t}
$$

$\forall m, j, t$,

$$
\sum_{l=1}^{n_{m}} S Q_{m l j t} \geq \sum_{l=1}^{n_{m}} C Q_{(m-1) l j t} \quad \forall j, t, m=2, \ldots, M
$$

$$
S Q_{m l j t} \geq C Q_{m l i t}+S_{i j m} . Y_{m l i j t}-B i g M .\left(1-Y_{m l i j t}\right)
$$$$
\forall m, l, i, j, t, i \neq j,
$$

$$
S Q_{m l j t} \leq U \cdot Q_{m l j t} \quad \forall m, l, j, t
$$

$S Q_{m l j t} \leq S O_{m l j t} \quad \forall m, l, j, t$,

$$
S Q_{m l j t} \geq S O_{m l j t}-U \cdot\left(1-Q_{m l j t}\right) \quad \forall m, l, j, t,
$$

$$
C Q_{m l j t} \leq U^{\prime} \cdot Q_{m l j t} \quad \forall m, l, j, t,
$$

$$
\begin{aligned}
& C Q_{m l j t} \leq C O_{m l j t} \quad \forall m, l, j, t, \\
& C Q_{m l j t} \geq C O_{m l j t}-U^{\prime} .\left(1-Q_{m l j t}\right) \quad \forall m, l, j, t, \\
& X Q_{m l j t} \leq U^{\prime \prime} \cdot Q_{m l j t} \quad \forall m, l, j, t, \\
& X Q_{m l j t} \leq X_{j m t} \quad \forall m, l, j, t, \\
& X Q_{m l j t} \geq X_{j m t}-U^{\prime \prime} \cdot\left(1-Q_{m l j t}\right) \quad \forall m, l, j, t, \\
& X Q_{m l j t}, S Q_{m l j t}, C Q_{m l j t} \geq 0 \quad \forall m, l, j, t,
\end{aligned}
$$

where the values of $U, U^{\prime}$, and $U^{\prime \prime}$ are the upper bounds of variables $S O_{m l j t}, C O_{m l j t}$, and $X_{j m t}$. The numbers of additional continuous variables and constraints are added to the model equal to $3 N . T . \sum_{m=1}^{M} n_{m}$ and $9 N . T . \sum_{m=1}^{M} n_{m}$, respectively.

In Table 2, benchmark test on the complexity (in terms of the number of binary variables, continuous variables, and constraints) of the proposed linearized model with respect to the former models is conducted. Table 3 shows numerical examples that compare former and proposed models in specific problem sizes with $\left(N, M, n_{m}, T\right)=(5,3,2,6)$ and $\left(N, M, n_{m}, T\right)=$ $(25,5,3,12)$. As can be observed from Table 4, even with the addition of some continuous variables and a number of constraints to the linearization, the complexity of the proposed model is significantly less than that of the second modeling approach proposed by Mohammadi and Jafari [24].

Tables 1-4 show that the new model is superior to the former models. The new model reduces the computational complexity and, furthermore, determines the starting and completion times of each product on each machine in each period of planning. Thus, it can be useful for considering the scheduling objectives in the planning.

Table 3. A comparison between former models and the proposed linearized model.

\begin{tabular}{cccc}
\hline Criterion & $\begin{array}{c}\text { Mohammadi's } \\
\text { model [20] }\end{array}$ & $\begin{array}{c}\text { Mohammadi \& } \\
\text { Jafari's } \\
\text { model [24] }\end{array}$ & Proposed model \\
\hline $\begin{array}{c}\text { Number of } \\
\text { binary variables }\end{array}$ & $3 N^{2} T(N+2) \sum_{m=1}^{N} n_{m}$ & $N^{3} T \sum_{m=1}^{M} n_{m}$ & $N T\left(M+(1+N) \sum_{m=1}^{M} n_{m}\right)$ \\
Number of & $N T\left(M+6 N \sum_{m=1}^{M} n_{m}\right)$ & $N T\left(M+2 N \sum_{m=1}^{M} n_{m}\right)$ & $N T\left(2 M+5 \sum_{m=1}^{M} n_{m}\right)$ \\
continuous \\
variables
\end{tabular}


Table 4. A comparison between former models and the proposed linearized model for two different problem sizes.

\begin{tabular}{|c|c|c|c|c|c|c|}
\hline & \multicolumn{3}{|c|}{$N=5, M=3, n_{m}=2, T=6$} & \multicolumn{3}{|c|}{$N=25, M=5, n_{m}=3, T=12$} \\
\hline & $\begin{array}{c}\text { Mohammadi \& } \\
\text { Jafari's } \\
\text { model }[24]\end{array}$ & $\begin{array}{c}\text { Proposed } \\
\text { model }\end{array}$ & Difference & $\begin{array}{c}\text { Mohammadi \& } \\
\text { Jafari's } \\
\text { model }[24]\end{array}$ & $\begin{array}{c}\text { Proposed } \\
\text { model }\end{array}$ & Difference \\
\hline Number of binary variables & 4,500 & 1,170 & 3,330 & $2,812,500$ & 118,500 & $2,694,000$ \\
\hline Number of continuous variables & 1,890 & 990 & 900 & 226,500 & 22,500 & 204,000 \\
\hline Number of constraints & 9,027 & 3,405 & 5,622 & $7,099,445$ & 170,825 & $6,928,620$ \\
\hline
\end{tabular}

\section{Development of heuristic algorithms based on rolling horizon}

Solving the single-stage multi-item multi-period CLSP with sequence-dependent setups is equivalent to solving multiple dependent Travelling Salesman Problems (TSPs) [38]. Hence, like the TSP, the CLSP belongs to the so called NP-hard problems. That means it is very difficult to optimally solve large instances of the problem. The introduction of multi-stage production with parallel machines makes the problem even more complicated. Therefore, it is necessary to find reasonable heuristic methods for medium and large-size instances [23].

Similar to Mohammadi [23] and Mohammadi and Jafari [24], rolling-horizon heuristics have been used to solve this problem.

\subsection{Idea}

Since manufacturing environments are dynamic, the quantities of production should be continually updated in response to disturbances such as delay in the delivery of raw materials, equipment failure, demand changes, etc. Rolling-horizon heuristic is one of the appropriate approaches that have been suggested in the literature for solving dynamic lot-sizing and scheduling problem, where demands are gradually revealed during the planning horizon. While the estimation of the demands of the early periods is more precise, the demands of the later periods are approximate. If in the integrated production planning and scheduling problem there is a subset of early periods that includes detailed scheduling decisions, and the late planning periods only include high-level decisions (i.e., production quantities), or relaxation formulation and aggregation modeling are used for these periods, the computational complexity will decrease. Production quantities for earlier periods are exact and can be used directly for the production process, while the quantities obtained for late periods (as rolling periods) are updated. As mentioned before, production quantities are continually updated; therefore, using the approximate solutions for the late periods affects the quality of final solution significantly.

Using rolling horizon heuristics for large-size MIP problems greatly reduces the computational complex- ity by substituting most of the binary variables and constraints with continuous variables and constraints. Therefore, this approach is still useful when all parameters are completely known. This approach initially decomposes the model into a set of smaller MIP models, each with a more tractable and smaller number of binary variables, and the decision variables are then iteratively determined [2,18-20,23-26,39].

According to the presented framework by Merce and Fontan [39], each iterative procedure decomposes the planning horizon into three parts. For a specified iteration $k$ :

- The first section (initial section) consists of $(k-1)$ periods. In this section, according to the previous iterations of the algorithm, the decision variables have been partially or completely frozen based on the selected freezing strategy;

- The second section (central section) only includes the $k$ th planning period. In this section, the whole problem is considered. In this section, all binary variables that are associated with this planning period are accounted in the model as binary forms;

- The third section (ending section) includes the remaining periods (from period $k+1$ to period $T$ ), which is simplified according to the chosen simplification strategy. Considering the degree of complexity reduction in the final section according to the selected simplification method, the larger-size problems are solved at reasonable computational times.

At the end of each iteration, $k$, one period is rolled for entering all sections of the algorithm into the new iteration. The mentioned procedure is terminated when iteration $T$ is carried out and the planning horizon comes to an end. The last iteration of the algorithm determines all decision variables in the overall planning horizon. Figure 1 demonstrates the MIP-based iterative procedure based on rolling horizon.

\subsection{Heuristic algorithms}

In this paper, three heuristics based on the iterative framework mentioned above are used to solve the 


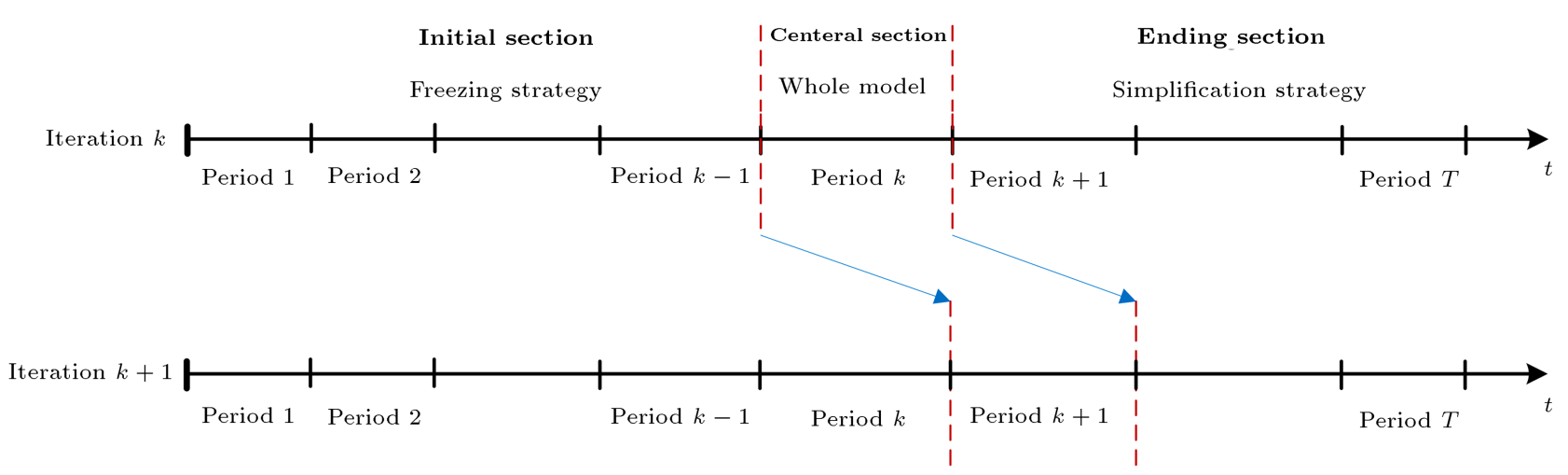

Figure 1. Illustration of the iterative procedure.

studied problem. These three MIP-based heuristics are based on iterative resolutions of reduced-size MIPs and rolling horizon. The steps of heuristic Algorithm 1 are as follows:

\section{- Heuristic Algorithm 1 (HA1)}

- Initial section: All decision variables and constraints are frozen over the beginning section.

- Central section: Consists of one period, the whole problem is considered.

- Ending section: Binary variables are relaxed for the ending section.

The simplification strategy chosen for the ending section ignores setup mechanisms from period $k+1$ to period $T$. In each iteration, the only binary variables involved in the model are related to the central section; thus, the computational effort required for solving the problem is considerably reduced and, hence, it permits to solve larger instances of the problem. The solution procedure initially decomposes the model into a succession of $T$ smaller MIPs, each with a smaller number of binary variables. We should note that the beginning section does not exist for the first iteration.

- Heuristic Algorithm 2 (HA2). The only difference between heuristics HA1 and HA2 is in their corresponding freezing policies in the initial section. In Heuristic Algorithm 2, only binary variables related to the initial section are frozen. Both the central and the ending sections for this heuristic are similar to those for the Heuristic Algorithm 1 (HA1).

- Heuristic Algorithm 3 (HA3). As mentioned above, the whole problem is considered for the central section. Thus, each iteration of the former heuristics (HA1 and HA2) has $N \cdot\left(M+(1+N) \cdot \sum_{m=1}^{M} n_{m}\right)$ binary variables in its central section. By increasing the size of the problem (product, machine, and stage number), the time taken to solve the MIP model in the central section tends to explode exponentially and a faster solution approach is needed to make decisions by solving a succession of smaller MIPs. An approach that can be used to solve larger instances of the problem in the central section is fix-and-relax method [40].

In Heuristic Algorithm 3 (HA3), the search space is limited by fix-and-relax method. This procedure involves solving a series of partially relaxed MIP models. For each MIP model, the number of binary variables is small enough to be quickly and optimally solved by exact solution methods such as branch-andbound, branch-and-cut, etc. As the series progresses, binary variables are permanently set at their obtained solution values and the number of relaxed variables is reduced. Speed is a major advantage of fix-and-relax method $[23,19]$.

The fix-and-relax procedure performed in period (iteration) $k$ related to the central section contains the following steps:

- Step 1. To identify the product sequence at stage 1, solve the simplified MIP model using partial linear programming relaxation, where the values of $Y_{1 l i j k}$ are constrained to be 0 or 1 .

- Step 2. For $m=2, \ldots, M$, solve the partial linear programming relaxation with $Y_{1 l i j k}$ fixed at their 0 or 1 solution values obtained from Step 1, $Y_{2 l i j k}$ to $Y_{(M-1)_{l i j k}}$ fixed at their 0 or 1 solution values from the previous applications of Step 2, and $Y_{M l i j k}$ constrained to be 0 or 1 while the remaining $Y$ variables may vary continuously between 0 and 1 .

Each cycle of Steps 1 and 2 of the mentioned fixand-relax method involves solving $M$ problems with just $N^{2} \cdot \sum_{m=1}^{M} n_{m}$ binary variables. Due to Constraint sets (10) to (12), less than $N-1$ binary variables in each problem are equal to 1 . In other words, more than $N \cdot\left(N \cdot \sum_{m=1}^{M} n_{m}-1\right)$ of $N^{2} \cdot \sum_{m=1}^{M} n_{m}$ binary variables in each problem will newly be 0 due to those constraints. Thus, the application of the cyclic fix-andrelax approach involves the solution to $M$ and $T$ MIPs with $N^{2} \cdot \sum_{m=1}^{M} n_{m}$ binary variables. 
Table 5. A comparison between HA1, HA2, and HA3.

\begin{tabular}{|c|c|c|c|}
\hline & HA1 & HA2 & HA3 \\
\hline Number of iterations & $T$ & $T$ & M.T \\
\hline $\begin{array}{c}\text { Number of binary } \\
\text { variables in each iteration }\end{array}$ & $N\left(M+(1+N) \sum_{m=1}^{M}\right)$ & $N\left(M+(1+N) \sum_{m=1}^{M} n_{m}\right)$ & $N^{2} \sum_{m=1}^{M} n_{m}$ \\
\hline
\end{tabular}

As mentioned earlier, in each MIP of heuristics HA1 and HA2, there exists $N \cdot\left(M+(1+N) \cdot \sum_{m=1}^{M} n_{m}\right)$ binary variables and in each MIP of heuristic HA3, there exists $N^{2} \cdot \sum_{m=1}^{M} n_{m}$ binary variables. Therefore, Heuristic Algorithm 3 (HA3) is able to solve instances with larger sizes. A comparison between heuristics has been shown in Table 5 .

\section{Computational results}

In previous sections, the new modeling for integrated lot-sizing and scheduling problem in a flexible flow shop environment has been formulated successfully, and the comparison of this model with the former models points out the higher efficiency of the proposed model. Due to the inherent complexity of the considered problem, small-size problems can be solved exactly by using optimization approaches such as "branch and bound," "branch and cut," etc. Therefore, the presented rollinghorizon heuristics have been used to solve this problem. This section provides the computational experiments used to evaluate the performance of the heuristics in finding good-quality solutions.

To evaluate and compare the performance of the heuristics, 20 problems with different sizes were selected. Similar to Mohammadi [23] and Mohammadi and Jafari [24], the required parameters are extracted from the following uniform distribution:

$$
\begin{aligned}
& b_{j m} \approx U(1.5,2), \quad d_{j t} \approx U(0,180), \\
& h_{j m} \approx U(0.2,0.4), \quad p_{j m t} \approx U(1.5,2), \\
& w_{i j m} \approx U(35,70), \quad S_{i j m} \approx U(35,70) \\
& \text { and } C_{m t} \approx U(a, b), \quad a=200 N+100(m-1), \\
& b=200 N+200(m-1) .
\end{aligned}
$$

$C_{m t}$ is calculated in a way to satisfy demands of each period on a just-in-time basis with average setups. For every problem size, five instances were randomly generated using the uniform distributions mentioned above, and the average results were recorded as a performance criterion for the heuristics. The number of jobs, machines, stages, and periods have profound influence on computational complexity of the problem.

The proposed model and lower MIP-based heuristics are coded in GAMS IDE (version 23.6.5) software

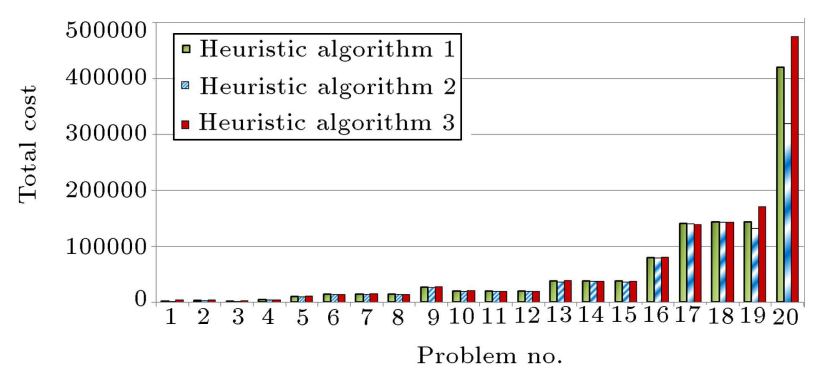

Figure 2. Comparative results obtained by the heuristics for the test problems.

and solved using GLPK GAMS module. All tests are conducted on an Intel corei2 laptop at $2.1 \mathrm{GHz}$ with 2GB of RAM.

The average results obtained from solving the 20 mentioned problems have been listed in Table 6 . Table 6 compares the objective function values and the run times spent for solving the problems by the heuristic methods. The "O.V." column indicates the objective values (total costs) resulting from the specified heuristic method. The lower objective value shows better performance of the solution methods. Figure 2 shows comparative results obtained by the heuristics for the test problems.

As can be seen from Table 6, all three heuristics can solve all 20 test problems in less than 3600 seconds. As it is expected, Heuristic Algorithm 1 solves the problem faster than both solution methods HA2 and HA3. Also, HA1 can obtain the minimum makespan for small-size instances. Both algorithms HA1 and HA2 can reach a good quality solution in reasonable time and the objective values obtained by HA1 and HA 2 are close to each other, also better than HA3 results. The computational time required to solve problems with HA1 is smaller than that with both HA2 and HA3 heuristics. For small and medium-size test problems, HA3 algorithm has relatively the same results with other methods. But, its results will be worse as the problem size increases.

As can be seen from Table 6 and Figure 2, the mean objective value for the heuristic HA2 is less than that obtained by the other heuristics HA1 and HA3. Thus, HA2 has a better performance for this problem. But, the HA2 needs more computational time to solve these test problems compared to HA1. Thus, HA2 is superior for the mentioned problem.

On average, the results obtained by heuristic HA2 are $10.54 \%$ and $17.96 \%$ better than the results of other 
Table 6. Comparative results obtained by the heuristics.

\begin{tabular}{|c|c|c|c|c|c|c|c|}
\hline \multirow{2}{*}{$\begin{array}{c}\text { Prob. } \\
\text { no. }\end{array}$} & \multirow{2}{*}{$\begin{array}{c}\text { Size } \\
\left(N \cdot M \cdot n_{m} \cdot T\right)\end{array}$} & \multicolumn{2}{|c|}{ Heuristic algorithm 1} & \multicolumn{2}{|c|}{ Heuristic algorithm 2} & \multicolumn{2}{|c|}{ Heuristic algorithm 3} \\
\hline & & O.V. & CPU time (s) & O.V. & CPU time (s) & O.V. & CPU time (s) \\
\hline 1 & $3 \times 3 \times 2 \times 3$ & $1759.2^{*}$ & 0.23 & 2121.6 & 0.25 & 3107.8 & 1.09 \\
\hline 2 & $5 \times 3 \times 2 \times 3$ & 3370.2 & 0.34 & 3370.2 & 0.33 & 3370.2 & 1.05 \\
\hline 3 & $3 \times 5 \times 2 \times 3$ & 1229.6 & 0.44 & 2087.9 & 0.39 & 2948.4 & 1.99 \\
\hline 4 & $3 \times 3 \times 2 \times 5$ & 4089.0 & 0.46 & 4083.1 & 0.46 & 4083.1 & 1.79 \\
\hline 5 & $5 \times 5 \times 2 \times 5$ & 10328.6 & 0.98 & 10281.3 & 0.64 & 10281.3 & 3.19 \\
\hline 6 & $7 \times 5 \times 2 \times 5$ & 13792.0 & 1.37 & 13717.1 & 1.32 & 13717.1 & 3.15 \\
\hline 7 & $5 \times 7 \times 2 \times 5$ & 14415.2 & 0.76 & 14415.2 & 0.78 & 14333.7 & 4.20 \\
\hline 8 & $5 \times 5 \times 2 \times 7$ & 14752.6 & 1.32 & 14473.5 & 1.14 & 14181.0 & 4.42 \\
\hline 9 & $7 \times 7 \times 2 \times 7$ & 27023.5 & 3.93 & 26794.0 & 2.76 & 26727.6 & 10.20 \\
\hline 10 & $10 \times 5 \times 2 \times 5$ & 20344.5 & 2.51 & 19585.1 & 2.30 & 20344.5 & 9.02 \\
\hline 11 & $5 \times 10 \times 2 \times 5$ & 19633.8 & 1.22 & 19633.8 & 1.19 & 19633.8 & 6.94 \\
\hline 12 & $5 \times 5 \times 2 \times 10$ & 19985.5 & 2.11 & 19622.3 & 1.60 & 19622.3 & 6.86 \\
\hline 13 & $10 \times 7 \times 2 \times 7$ & 38490.6 & 8.78 & 35993.0 & 8.05 & 38289.3 & 37.52 \\
\hline 14 & $7 \times 10 \times 2 \times 7$ & 37643.7 & 4.80 & 37626.6 & 6.08 & 37600.4 & 24.65 \\
\hline 15 & $7 \times 7 \times 2 \times 10$ & 37511.4 & 7.28 & 37061.4 & 8.28 & 36814.5 & 22.97 \\
\hline 16 & $10 \times 10 \times 2 \times 10$ & 80029.8 & 14.22 & 80029.8 & 15.21 & 80029.8 & 547.53 \\
\hline 17 & $15 \times 10 \times 2 \times 10$ & 139984.3 & 34.12 & 139984.3 & 37.65 & 138316.4 & 1437.56 \\
\hline 18 & $10 \times 15 \times 2 \times 10$ & 143168.9 & 27.16 & 143168.9 & 27.11 & 143148.6 & 1875.00 \\
\hline 19 & $10 \times 10 \times 2 \times 15$ & 142698.4 & 32.06 & 132698.4 & 34.00 & 169843.5 & 2153.36 \\
\hline \multirow[t]{2}{*}{20} & $15 \times 15 \times 2 \times 15$ & 420215.2 & 195.57 & 320215.2 & 191. 89 & 473957.8 & 3600.00 \\
\hline & Average & 59523.30 & 16.98 & 53848.14 & 17.07 & 63517.57 & 487.62 \\
\hline
\end{tabular}

* Bold items show the minimum makespan obtained by the solution methods.

heuristics HA2 and HA3, respectively:

$$
\begin{aligned}
& \left(\frac{59523.30-53848.14}{53848.14}\right) \times 100=10.54 \% \\
& \left(\frac{63517.57-53848.14}{53848.14}\right) \times 100=17.96 \% .
\end{aligned}
$$

- Sensitivity analysis. Here, sensitivity analysis is used to clarify the problem and ensure the suitability of the proposed model. A sensitivity analysis is a technique used to determine how different values of an independent variable will affect a particular dependent variable under a given set of assumptions.

For sensitivity analysis, a problem instance with the size $\left(N . M . n_{m} \cdot T=10.5 .2 .12\right)$ is considered. In other words, in a flexible flow shop production system with 5 stages so that at every stage there are two identical parallel machines, we want to determine lot-size and sequence of 10 different products in a planning horizon with 12 periods. Data for the mentioned problem is generated randomly using a uniform distribution described in the previous section. With a change in the original value of available capacity for machines, we evaluate the impact of this parameter on the objective function
Table 7. The impact of change in available capacity $(\%)$ on the objective function.

\begin{tabular}{cccc}
\hline $\begin{array}{c}\text { Change in } \\
\text { available } \\
\text { capacity (\%) }\end{array}$ & $\begin{array}{c}\text { Ptoduction } \\
\text { cost }\end{array}$ & $\begin{array}{c}\text { Inventory } \\
\text { cost }\end{array}$ & $\begin{array}{c}\text { Total } \\
\text { cost }\end{array}$ \\
\hline$-50.00 \%$ & 33033.37 & 3825.18 & 47869.67 \\
$-37.50 \%$ & 32866.31 & 3844.36 & 47666.11 \\
$-25.00 \%$ & 32697.14 & 3914.09 & 47510.28 \\
$-12.50 \%$ & 32593.10 & 3921.65 & 47379.12 \\
$0.00 \%$ & 32472.33 & 3971.30 & 47267.74 \\
$12.50 \%$ & 32342.88 & 4047.95 & 47171.79 \\
$25.00 \%$ & 32210.42 & 4132.19 & 47079.42 \\
$37.50 \%$ & 32090.35 & 4211.47 & 46998.61 \\
$50.00 \%$ & 31990.38 & 4276.69 & 46930.52 \\
\hline
\end{tabular}

value. The parameter of available capacity increases and decreases by $50 \%$ of its original value. Heuristic method 2 (HA2) is used to solve the described problem and the obtained results are summarized in Table 7. As can be seen from Table 7, the total cost and production cost increase by increasing the available capacity while inventory cost decreases. As it is known, the possibility of more production in periods with lower production costs is attained 
by increasing the available capacity, which results in a decrease in production cost. Obviously, the inventory level will increase because of this increase in production amounts in the period under consideration, which in turn could increase inventory cost. The resultant reduced production cost and increasing inventory cost lead to reduction in total cost. If the available capacity is reduced to less than $50 \%$ of the current capacity, the algorithm is not possible to reach a feasible solution, because it is not possible to satisfy demand of the customers with this new capacity.

\section{Conclusion}

In this paper, integrated lot-sizing and scheduling problem in a multi-product, multi-period production system in a flexible flow shop with capacity constraint and sequence-dependent setup is addressed. The main contribution of the paper is presenting and testing a more efficient mathematical model to formulate the problem. In comparison to the models presented by Mohammadi [23] and Mohammadi and Jafari [24], the proposed model in this paper provides a more efficient formulation with remarkably less complexity (in terms of the number of binary variables, continuous variables, and constraints) for the problem. Due to the computational complexity of the problem, three heuristic methods based on mathematical modeling were used for solving the problem. In the same limited computational time, the numerical experiments showed that HA2 was superior for these problem instances. Because of the simplicity of the heuristic, it can be easily applied to the real production systems and largesize problems. A general review of the results shows that:

- All three solution methods can solve all the test problems in less than $3600 \mathrm{~s}$;

- The computational time required to solve problems with HA1 is smaller than those with both HA2 and HA3 heuristics;

- The mean objective value obtained by HA2 is better than the results of both HA1 and HA3. Therefore, HA2 is superior for the mentioned problem.

One straightforward area for future research is the development of the proposed model to include real conditions of manufacturing environments, such as limited intermediate buffer space, lot transportation constraints, etc. Considering the uncertainty in production system is one of the interesting areas for research. Also, considering the development of meta-heuristic approaches for solving combinatorial optimization problems, the application of metaheuristic approaches can be another area for future research.

\section{References}

1. Fandel, G. and Stammen-Hegene, C. "Simultaneous lot sizing and scheduling for multi-product multi-level production", Int. J. Prod. Econ., 104(2), pp. 308-316 (2006).

2. Ramezanian, R., Saidi-Mehrabad, M. and Teymouri, E. "A mathematical model for integrating lot-sizing and scheduling problem in capacitated flow shop environments", Int. J. Adv. Manuf. Technol., 66, pp. 347361 (2013).

3. Yan, H-S., Xia, Q-F., Zhu, M-R., Liu, X-L. and Guo, Z-M. "Integrated production planning and scheduling on automobile assembly lines", IIE Trans, 35(8), pp. 711-725 (2003).

4. Riane, F., Artiba, A. and Iassinovski, S. "An integrated production planning and scheduling system for hybrid flowshop organizations", Int. J. of Prod. Econ., 74, pp. 33-48 (2002).

5. Lee, I., Sikora, R. and Shaw, M.J. "A genetic algorithm-based approach to flexible flow-line scheduling with variable lot sizes", IEEE Trans Syst Man Cybern B Cybern, 27(1), pp. 36-54 (1999).

6. Wagner, H-M. and Whithin, T-M. "Dynamic version of the economic lot size model", Manage. Si., 5, pp. 89-96 (1958).

7. Karimi-Nasab, M. and Modarres, M. "Lot sizing and job shop scheduling with compressible process times: a cut and branch approach", Comput. Ind. Eng., 85, pp. 196-205 (2015).

8. Tempelmeier, H. and Buschkuhl, L. "Dynamic multimachine lotsizing and sequencing with simultaneous scheduling of a common setup resource", Int. J. Prod. Econ., 13(1), pp. 401-412 (2008).

9. Buschkühl, L., Sahling, F., Helber, S. and Tempelmeier, H. "Dynamic capacitated lot-sizing problems: A classification and review of solution approaches", OR Spekt, 32, pp. 231-261 (2010).

10. Kimms, A., Multi-Level, Single-Machine Lot Sizing and Scheduling (with Initial Inventory), Manuskripte aus den Instituten für Betriebswirtschaftslehre der Universität Kiel, No. 329, Kiel (1993).

11. Kimms, A. "Multi-level, single-machine lotsizing and scheduling (with initial inventory)", Eur. J. Oper. Res., 89(1), pp. 86-99 (1996).

12. Kimms, A. and Drexl, A. "Some insights into proportional lotsizing and scheduling", J. Oper. Res., 49(11), pp. 1196-1205 (1998).

13. Fleischmannand, B. and Meyr, H. "The general lotsizing and scheduling problem", Or Spekt, 19(2), pp. 11-21 (1997).

14. Ponnambalam, S.G. and Mohan Reddy, M. "A GA-SA multiobjective hybrid search algorithm for integrating lot sizing and sequencing in flow-line scheduling", Int. J. Adv. Manuf. Technol., 21, pp. 126-137 (2003). 
15. Toledo, C.F.M., Kimms, A., França, P.M. and Morabito, R. "The synchronized and integrated two-level lot sizing and scheduling problem: Evaluating the generalized mathematical model", Math. Prob. in Eng., pp. 1-18 (2015).

16. Mohammadi, M., Fatemi Ghomi, S-M-T., Karimi, B. and Torabi, S-A. "Rolling-horizon and fix-and-relax heuristics for the multiproduct multi-level capacitated lotsizing problem with sequencedependent setups", $J$. Intell. Manuf., 21(4), pp. 501-510 (2010).

17. Mohammadi, M., Fatemi Ghomi, S-M-T., Karimi, B. and Torabi, S.-A. "MIP-based heuristics for lotsizing in capacitated pure flow shop with sequence-dependent setups", Int. J. Prod. Res., 48(10), pp. 2957-2973 (2010).

18. Ramezanian, R., Saidi-Mehrabad, M. and Fattahi, P. "MIP formulation and heuristics for multi-stage capacitated lot-sizing and scheduling problem with availability constraints", J. of Manuf. Syst., 32, pp. 392-401 (2013).

19. Ramezanian, R., Saidi-Mehrabad, M. and Fattahi, P. "Integrated lot-sizing and scheduling with overlapping for multi-level capacitated production system", Int $J$. of Comp. Integ. Manuf., 26(7), pp. 681-695 (2013).

20. Ramezanian, R. and Saidi-Mehrabad, M. "Hybrid simulated annealing and MIP-based heuristics for stochastic lot-sizing and scheduling problem in capacitated multi-stage production system", Appl. Math. Model., 37, pp. 5134-5147 (2013).

21. Mohammadi, M., Torabi, S.-A., Fatemi Ghomi, S.M.-T. and Karimi, B. "A new algorithmic approach for capacitated lot-sizing problem in flow shops with sequence-dependent setups", Int. J. Adv. Manuf. Technol., 49, pp. 201-211 (2010).

22. Mohammadi, M., Fatemi Ghomi, S.-M.-T. and Jafari, N. "A genetic algorithm for simultaneous lotsizing and sequencing of the permutation flow shops with sequence-dependent setups", Int. J. of Comp. Integ. Manuf., 24(1) , pp. 87-93 (2011).

23. Mohammadi, M. "Integrating lotsizing, loading and scheduling decisions in flexible flow shops", Int. J. Adv. Manuf. Technol., 50, pp. 1165-1174 (2010).

24. Mohammadi, M. and Jafari, N. "A new mathematical model for integrating lot sizing, loading and scheduling decisions in flexible flow shops", Int. J. Adv. Manuf. Technol., 55, pp. 709-721 (2011).

25. Clark, A.-R. "Optimization approximations for capacity constrained material requirements planning", Int. J. Prod. Econ., 84(2), pp. 115-131 (2003).

26. Clark, A.-R. and Clark, S.-J. "Rolling-horizon lotsizing when setup times are sequence-dependent", Int. J. Prod. Res., 38(10), pp. 2287-2308 (2000).

27. Babaei, M., Mohammadi, M. and Fatemi Ghomi, S.M.T. "A genetic algorithm for the simultaneous lot sizing and scheduling problem in capacitated flow shop with complex setups and backlogging", Int J. Adv. Manuf. Technol., 70(1-4), pp. 125-134 (2013).
28. Urrutia, E.D.G., Aggoune, R. and Dauzère-Pérès, S. "Solving the integrated lot-sizing and job-shop scheduling problem", Int J. of Prod. Res., 52(17), pp. 5236-5254 (2014).

29. Wolosewicz, C., Dauzère-Pérès, S. and Aggoune, R. "A Lagrangian heuristic for an integrated lot-sizing and fixed scheduling problem", Eur. J. Oper. Res., 244(1), pp. 3-12 (2015).

30. Karimi-Nasab, M., Modarres, M. and Seyedhoseini, S. "A self-adaptive PSO for joint lot sizing and job shop scheduling with compressible process times", Appl. Soft. Comput., 27, pp. 137-147 (2015).

31. Karimi, B., Fatemi Ghomi, S.M.T. and Wilson. J.M. "The capacitated lot sizing problem: A review of models and algorithms", Omega, 31(5), pp. 365-378 (2003).

32. Yan, H-S., Wan, X-Q. and Xiong F-L. "Integrated production planning and scheduling for a mixed batch jobshop based on alternant iterative genetic algorithm", J. Oper. Res. Soci., 66(8), pp. 1250-1258 (2015).

33. Rohaninejad, M., Kheirkhah A. and Fattahi, P. "Simultaneous lot-sizing and scheduling in flexible job shop problems", Int. J. Adv. Manuf. Technol., 78(1), pp. 1-18 (2015).

34. Copil, K., Wörbelauer, M., Meyr, H. and Tempelmeier H. "Simultaneous lotsizing and scheduling problems: a classification and review of models", OR Spec, $\mathbf{3 9}(1)$, pp. 1-64 (2016).

35. Schemeleva, K., Delorme. X. and Dolgui, A. "A memetic algorithm for a stochastic lot-sizing and sequencing problem", IFAC-Papers OnLine, 48(3), pp. 1809-1814 (2015).

36. Masmoudi, O., Yalaoui, A., Ouazene, Y. and Chehade, H. "Lot-sizing in flow-shop with energy consideration for sustainable manufacturing systems", IFACPapersOnLine, 48(3), pp. 727-732 (2015).

37. Bisschop, J., AIMMS Optimization Modeling. Paragon Decision Technology, B.V., Harlem (2006).

38. Gupta, D. and Magnusson, T. "The capacitated lot sizing and scheduling problem with sequence-dependent setup costs and setup times", Comput. Operat. Res., 32, pp. 727-747 (2005).

39. Merece, C. and Fonton, G. "MIP-based heuristics for capacitated lotsizing problems", Int. J. Prod. Econ., 85(1), pp. 97-111 (2003).

40. Dillenberger, C., Escudero, L.F., Wollensak, A. and Zhang, W. "On practical resource allocation for production planning and scheduling with period overlapping setups", Eur. J. Oper. Res., 75(2), pp. 275-286 (1994).

\section{Biographies}

Reza Ramezanian received BSc, $\mathrm{MSc}$, and $\mathrm{PhD}$ degrees all in Industrial Engineering from Iran University of Science and Technology, Tehran, Iran, and is 
currently Assistant Professor of Industrial Engineering at K. N. Toosi University of Technology, Tehran, Iran, and is reviewer of several international journals. His research interests include operation research, stochastic optimization, supply chain management, production planning, dynamic lot-sizing, and scheduling theory and applications.

Sahar Fallah Sanami received her BSc degree in Industrial Engineering from Behshahr branch of Iran University of Science and Technology, Behshahr, Iran, and MS degree in Economics and Management from Sem- nan University, Semnan, Iran. Her research interests include strategic management, research methodology in management, decision making, operation research, and production planning and scheduling.

Vahid Mahmoodian received his BSc degree in Industrial Engineering from Tabriz University, Tabriz, Iran, in 2012. He graduated from Iran University of Science and Technology, Tehran, Iran, with MSc degree in the same field. His research interests are novel exact optimization methods, data mining, artificial intelligence, and supply chain. 